\title{
A Robust Metric for Soft-Output Detection in the Presence of Class-A Noise
}

\author{
Dario Fertonani, Student Member, IEEE, and Giulio Colavolpe, Member, IEEE
}

\begin{abstract}
Digital communications over channels impaired by impulse noise are considered. We first address the problem from an information-theoretical viewpoint, discussing the performance limits imposed by the channel model. Then, we describe and compare a couple of practical communication schemes employing powerful channel codes and iterative decoding, with focus on a very simple and robust detection scheme that does not require the estimation of the statistics of the impulse noise.
\end{abstract}

Index Terms-Impulse noise, soft-output detection, achievable information rate.

\section{INTRODUCTION}

$\mathbf{T}$ HE power delivery networks and some mobile radio scenarios are often characterized by interferences that exhibit a significant impulsive nature. Among the various statistical models for these phenomena, generally referred to as "impulse noise", the most widely used in the literature is the class-A model [1], which is adopted also in this letter. The performance of such systems is generally studied under the assumption of ideal knowledge of the statistical properties of the impulse noise [2]-[4]. These statistics, which are actually unknown to the receiver, can be effectively estimated [5], but the estimation algorithms unfortunately affect the complexity of the system and cannot properly cope with time-varying channels [5]-[8]. A blind approach, based on detection metrics that do not require the knowledge of the channel parameters nor their estimation, is thus of great interest.

We first resort to information-theoretical arguments and discuss the ultimate performance limits imposed by the channel, then we consider practical communication schemes employing powerful codes and iterative decoding [9], [10]. In particular, we propose a detection scheme that does not require to know nor to estimate the statistics of the impulse noise, and compare it with an ideal receiver that perfectly knows such statistics and with the soft-limiting receivers [11], which are usually considered as a reference benchmark for robust detection over class-A channels. These comparisons prove the effectiveness of the proposed solution, which performs practically as the ideal one and much better than the classical soft-limiting receivers.

\section{Channel Model}

A sequence $c_{1}^{K}=\left\{c_{k}\right\}_{k=1}^{K}$ of $M$-ary complex-valued symbols, possibly obtained by properly encoding a sequence

Paper approved by T. M. Duman, the Editor for Coding Theory and Applications of the IEEE Communications Society. Manuscript received April 12, 2007; revised October 8, 2007.

The authors are with the Department of Information Engineering, University of Parma, Viale G. P. Usberti 181/A, 43100 Parma, Italy (e-mail: fertonani@tlc.unipr.it; giulio@unipr.it).

This work was presented in part at the IEEE Global Communications Conference (GLOBECOM'07), Washington, DC, USA, November 2007.

Digital Object Identifier 10.1109/TCOMM.2009.0901.070041 of information bits, is linearly modulated and transmitted over an additive white Gaussian noise (AWGN) channel that also introduces impulse noise. ${ }^{1}$ Assuming ideal synchronization and absence of intersymbol interference, we can write the received samples as [1]

$$
y_{k}=c_{k}+n_{k} \quad, \quad k \in\{1,2, \ldots, K\}
$$

where $\boldsymbol{n}_{1}^{K}$ is a sequence of independent and identically distributed noise samples. At each time epoch $k$, the statistical properties of the sample $n_{k}$ are completely defined by the channel state $s_{k}$, which belongs to the set of the non-negative integers $\mathbb{N}$, and assumes the value $i \in \mathbb{N}$ with probability [1]

$$
P_{i}=\frac{e^{-A} A^{i}}{i !}
$$

where $A$ is a positive parameter characterizing the channel, generally referred to as "impulsive index". In particular, the sample $n_{k}$ is a complex circularly-symmetric Gaussian random variable with variance depending on $s_{k}$, so that the probability density function (PDF) of $n_{k}$ conditioned to $s_{k}$ can be written as [1]

$$
p\left(n_{k} \mid s_{k}=i\right)=\frac{1}{2 \pi \sigma_{i}^{2}} \exp \left\{-\frac{\left|n_{k}\right|^{2}}{2 \sigma_{i}^{2}}\right\} \quad, \quad i \in \mathbb{N}
$$

where $\sigma_{i}^{2}$ is the variance per component of the noise samples when $s_{k}=i$. Hence, the PDF of the generic sample $n_{k}$ results

$$
p\left(n_{k}\right)=\sum_{i=0}^{\infty} P_{i} p\left(n_{k} \mid s_{k}=i\right)=\sum_{i=0}^{\infty} \frac{P_{i}}{2 \pi \sigma_{i}^{2}} \exp \left\{-\frac{\left|n_{k}\right|^{2}}{2 \sigma_{i}^{2}}\right\} .
$$

The variances $\left\{\sigma_{i}^{2}\right\}$ can be written as

$$
\sigma_{i}^{2}=\left(1+\frac{i}{A \Gamma}\right) \sigma_{0}^{2} \quad, \quad i \in \mathbb{N}
$$

where $\sigma_{0}^{2}$ can be interpreted as the variance per component of the background Gaussian noise, while $\Gamma$ is a positive parameter describing the power of the impulse noise [1]. Namely, since the average power of the noise samples is

$$
\mathbb{E}\left\{\left|n_{k}\right|^{2}\right\}=2 \sum_{i=0}^{\infty} P_{i} \sigma_{i}^{2}=2 \sigma_{0}^{2}+\frac{2 \sigma_{0}^{2}}{\Gamma}
$$

the channel introduces, in addition to the background Gaussian noise with average power $2 \sigma_{0}^{2}$, an impulsive contribution with average power $2 \sigma_{0}^{2} / \Gamma$.

By properly setting the values of the parameters $A$ and $\Gamma$, a large variety of channels with different statistical properties can be described [1], [2]. In this work, we focus on scenarios where the presence of impulsive noise, that is the event $\left\{s_{k}>0\right\}$, is relatively infrequent with respect to the

\footnotetext{
${ }^{1}$ For any sequence $\left\{v_{k}\right\}$, we denote the subsequence $\left\{v_{k}\right\}_{k=n_{1}}^{n_{2}}$ by $\boldsymbol{v}_{n_{1}}^{n_{2}}$.
} 




Fig. 1. Signal-to-noise ratio required to achieve an information rate of 1 bit per channel use.

presence of background noise only, that is the event $\left\{s_{k}=0\right\}$. Hence, we assume that $P_{0}>1 / 2$, or, equivalently, that the parameter $A$ satisfies the inequality

$$
A<\log _{e}(2) \simeq 0.693 \text {. }
$$

On the other hand, no particular restriction on the value of $\Gamma$ is assumed.

\section{Theoretical Performance Limits}

Before describing practical communication systems, we analyze the ultimate performance limits imposed by the channel. In particular, we are interested in evaluating the maximum number of information bits that can be transmitted per channel use, on average, to achieve an arbitrarily small bit-error rate (BER) when no upper limit on the length of $\boldsymbol{c}_{1}^{K}$ is imposed. This corresponds to evaluating the information rate $I(C, Y)$ between the sequences $\boldsymbol{c}_{1}^{K}$ and $\boldsymbol{y}_{1}^{K}$ [12]. We will restrict ourselves to the case of a stationary source and symbols $\boldsymbol{c}_{1}^{K}$ belonging to an $M$-ary phase-shift keying (PSK) alphabet. In this case, by resorting to the arguments in [13] or simply by exploiting the symmetry of both the channel and the alphabet, it is easy to prove that a memoryless source that emits equally likely symbols achieves the maximum allowed information rate. Such a source is thus considered hereafter. Although, for the considered system, the information rate $I(C, Y)$ cannot be written in a closed-form expression, we can easily evaluate it by numerical integration, exploiting the memoryless nature of both the source and the channel [12]. Some significant outcomes of such computations are reported and discussed in the following.

In Fig. 1, it is shown how the value of the signal-to-noise ratio (SNR) required to achieve an information rate of 1 bit per channel use varies when different channels are considered and a quaternary PSK (QPSK) modulation is adopted. In this letter, we define the SNR as $\left|c_{k}\right|^{2} /\left(2 \sigma^{2}\right)$, that is with respect to the background Gaussian noise only, so that the impulse noise is not involved in the definition. Together with the curves related to class-A channels, we also reported the corresponding curves related to the AWGN channel and to a system with ideal channel-state information (CSI), that is a system that knows

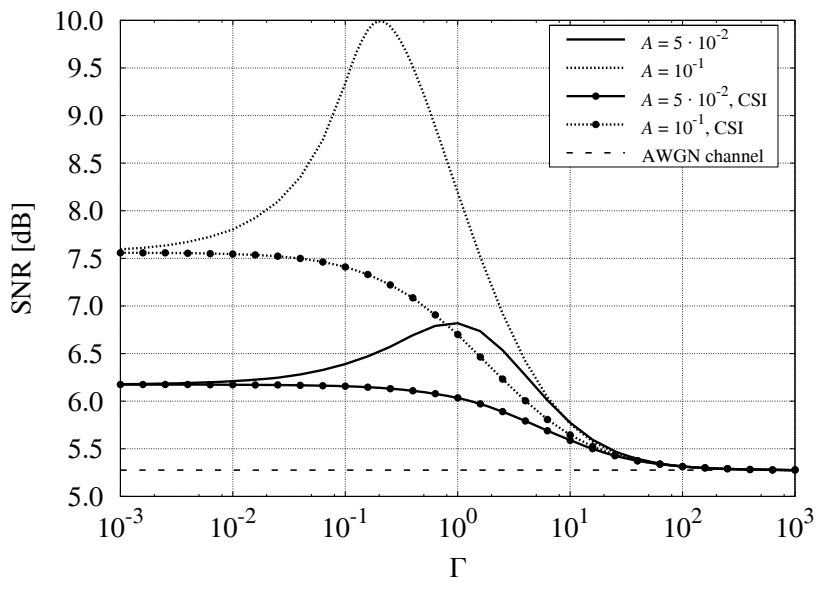

Fig. 2. Signal-to-noise ratio required to achieve an information rate of 1.75 bits per channel use.

the actual realization of the state process $s_{1}^{K}$ underlying the class-A channel. ${ }^{2}$ All following considerations qualitatively hold irrespectively of the values of the impulsive index and the target information rate, but, as shown in Fig. 2, the differences between the various performance limits are more significant as these values increase-when the impulsive index is small enough, all curves collapse on that related to the AWGN channel. It is interesting to note that the curves related to classA channels without CSI exhibit a non-monotonic behavior with respect to $\Gamma$. In particular, there exists a value $\Gamma_{0}$ such that, for $\Gamma<\Gamma_{0}$, the lower the value of $\Gamma$ the better the performance, up to an asymptotic value worsening as the impulsive index and/or the target information rate increase. This behavior, which is somehow surprising since it implies that the system can take advantage of a larger power of the impulsive interferers, ${ }^{3}$ can by explained by considering that the gap between the CSI system and the real system is due to a non-ideal channel-state identification. In fact, the curves related to CSI systems exhibit a monotonic behavior with respect to $\Gamma$, proving that the theoretical power efficiency worsens as the power of the impulse noise increases when ideal channel-state identification is available, as expected. This allows us to conjecture that the beneficial effect of an increasing power of the impulse noise is due to the fact that, when it is large enough, the system can better detect the presence of interfering impulses and, consequently, better approach the performance of the CSI system. Such conjectures are confirmed by the fact that the gap between real systems and CSI systems tends to vanish as the value of $\Gamma$ tends to zero, that is when the impulse noise is much more powerful than the background noise and thus is easier to detect.

\footnotetext{
${ }^{2}$ The information rate of CSI systems equals the statistical average of the information rates over the channel states [13].

${ }^{3}$ Although similar conclusions are drawn in [4] while discussing the numerical simulations, our results prove that the non-monotonic behavior is not due to any particular coding scheme, but is instead an intrinsic feature of the channels affected by impulse noise.
} 


\section{Practical Communication Schemes}

\section{A. Optimal Detection}

In a typical environment, the statistics of the impulse noise are such that, when no channel encoding is adopted, the system is basically impulse-noise limited and an error floor in the BER curve occurs [2]-[4]. We thus consider a communication system that adopts a powerful channel code, such as turbolike or low-density parity-check (LDPC) codes, and performs iterative decoding [9], [10]. For each time epoch $k$ and for each trial value $\tilde{c}_{k}$ belonging to the modulation alphabet, the optimal detector should send to the decoder a likelihood message $I_{k}\left(\tilde{c}_{k}\right)$, simply referred to as "metric" in the following, such that ${ }^{4}$

$$
I_{k}\left(\tilde{c}_{k}\right) \propto p\left(y_{k} \mid \tilde{c}_{k}\right)=\sum_{i=0}^{\infty} \frac{P_{i}}{2 \pi \sigma_{i}^{2}} \exp \left\{-\frac{\left|y_{k}-\tilde{c}_{k}\right|^{2}}{2 \sigma_{i}^{2}}\right\}
$$

where $p\left(y_{k} \mid \tilde{c}_{k}\right)$ is the PDF of the received sample $y_{k}$ conditioned to the transmission of the symbol $\tilde{c}_{k}$. In the case at hand, after straightforward manipulations of (8) based on (2) and (5), we can write the generic metric $I_{k}\left(\tilde{c}_{k}\right)$ as

$$
\begin{aligned}
I_{k}\left(\tilde{c}_{k}\right)= & \exp \left\{-\frac{\left|y_{k}-\tilde{c}_{k}\right|^{2}}{2 \sigma_{0}^{2}}\right\}+ \\
& \sum_{i=1}^{\infty} \frac{A^{i}}{i !} \frac{A \Gamma}{(A \Gamma+i)} \exp \left\{-\frac{\left|y_{k}-\tilde{c}_{k}\right|^{2}}{2 \sigma_{0}^{2}} \frac{A \Gamma}{A \Gamma+i}\right\}
\end{aligned}
$$

pointing out that the first term is exactly the optimal metric for AWGN channels, whereas the second term can be seen as a correction term accounting for the presence of impulse noise. The summation in (9) involves an infinite series, and thus it is not suitable for practical uses. Hence, we define the parameter $i_{\mathrm{MAX}}$ as the minimum integer such that

$$
\sum_{i=i_{\mathrm{MAX}}+1}^{\infty} P_{i}<10^{-10}
$$

and, slightly abusing the notation, we refer to the metric obtained by neglecting the terms with index $i>i_{\text {MAX }}$ in (9) as "optimal metric". When the hypothesis (7) is satisfied, we get $i_{\mathrm{MAX}} \leq 10$ irrespectively of the value of the impulsive index $A$.

\section{B. Suboptimal Detection}

According to (9), the evaluation of the optimal metrics requires that the receiver knows the values of $\sigma_{0}^{2}, A$ and $\Gamma$. Since these parameters are actually unknown to the receiver, the optimal metric can be just considered as an ideal solution. A possible practical approach consists of estimating the statistics of the noise by means of proper algorithms [5]. Since the estimation of the power of the background Gaussian noise is not critical, a perfect knowledge of the value of $\sigma_{0}^{2}$ is assumed in this letter. On the other hand, the algorithms for the estimation of the values of $A$ and $\Gamma$, besides increasing the complexity of the receiver, cannot properly cope with impulse noise whose statistics are significantly time-varying [5]. Hence, the design of detection schemes that do not require the knowledge of the

\footnotetext{
${ }^{4}$ We use the proportionality symbol $\propto$ when the sides can differ by a positive multiplicative factor irrelevant for the decoding process.
}

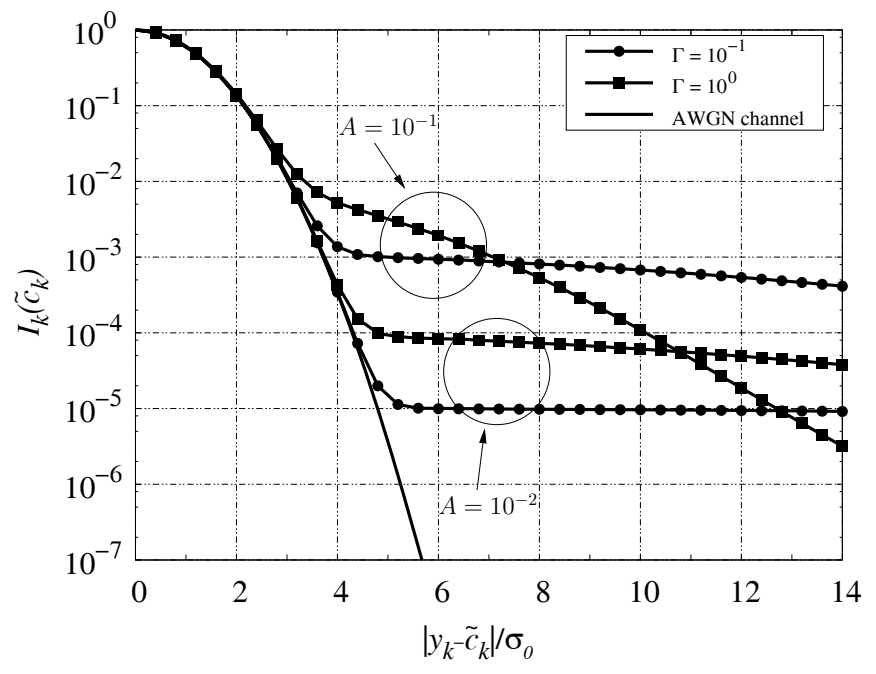

Fig. 3. Optimal metric for different parametrizations of the impulse noise.

values of $A$ and $\Gamma$ nor their estimation is of great interest for their simplicity and robustness [6]-[8]. A couple of possible solutions are compared in the following. ${ }^{5}$

We first consider the classical "soft limiting" (SL) metric [11]

$$
I_{k}^{\mathrm{SL}}\left(\tilde{c}_{k}\right)=\exp \left\{-\frac{\left|z_{k}-\tilde{c}_{k}\right|^{2}}{2 \sigma_{0}^{2}}\right\}
$$

where, at each time epoch $k$, the sample $z_{k}$ is obtained by properly cutting the amplitude of the received sample $y_{k}$. Formally, given a suitable threshold value $V_{T}>0$, we can write

$$
z_{k}=f\left(\Re\left\{y_{k}\right\}\right)+j f\left(\Im\left\{y_{k}\right\}\right)
$$

where the non-linear function $f(\cdot)$ is defined as

$$
f(x)=\left\{\begin{array}{ll}
x & \text { if }|x| \leq V_{T} \\
V_{T} & \text { if } x>V_{T} \\
-V_{T} & \text { if } x<-V_{T}
\end{array} .\right.
$$

The rationale of the SL metric is discussed in [11].

In this letter, we propose an alternative solution, basically extending our proposal in [7]. In Fig. 3, the behavior of the optimal metric $I_{k}\left(\tilde{c}_{k}\right)$ is shown as a function of $\left|y_{k}-\tilde{c}_{k}\right|$, for different values of $A$ and $\Gamma$. Under the hypothesis (7), the dominant term in (9) is the first one when the value of the Euclidean distance $\left|y_{k}-\tilde{c}_{k}\right|$ is low, so that all curves match the classical AWGN metric

$$
I_{k}^{\mathrm{AWGN}}\left(\tilde{c}_{k}\right)=\exp \left\{-\frac{\left|y_{k}-\tilde{c}_{k}\right|^{2}}{2 \sigma_{0}^{2}}\right\}
$$

at the left side of Fig. 3, irrespectively of the actual statistics of the impulse noise. On the other hand, at the right side of Fig. 3, that is for large values of $\left|y_{k}-\tilde{c}_{k}\right|$, the second term is the dominant one in (9) and the curves thus differ depending on the statistics of the impulse noise. Hence, in that region, any metric that is blind with respect to the values of $A$ and $\Gamma$ cannot approximate with accuracy the ideal metric. We exploit the fact that the knowledge of the values of $A$ and $\Gamma$ is

\footnotetext{
${ }^{5}$ Other solutions addressed to a different model of the impulse noise are presented in [6], but they are not considered here since they are not suitable for receivers employing iterative decoding [7], [8]
} 
required only for the description of the "tails" of the metric, and propose the following threshold approximation

$$
I_{k}\left(\tilde{c}_{k}\right)=\max \left\{I_{k}^{\mathrm{AWGN}}\left(\tilde{c}_{k}\right), \Delta\right\}
$$

where $\Delta \in[0,1]$ is a design parameter discussed later. The rationale of the approximation (15), which is just a saturation of the AWGN metric (not of the received sample, unlike the SL metric) to a constant threshold and thus is very simple from a computational viewpoint, is explained in the following. First, when the Euclidean distance $\left|y_{k}-\tilde{c}_{k}\right|$ is low, the proposed metric matches the AWGN metric, as an effective metric should definitely do according to Fig. 3. On the other hand, when the received sample is far away from the constellation, that is when the Euclidean distance $\left|y_{k}-\tilde{c}_{k}\right|$ is very large for all possible values of the modulation symbol $\tilde{c}_{k}$, the proposed metric is saturated to the minimum threshold $\Delta$ irrespectively of the value of $\tilde{c}_{k}$, so that the detector produces the so-called "erasure" decision. In this case, the presence of impulsive contributions in addition to the background noise is very likely, and a receiver that does not know the statistics of the impulse noise cannot produce any more reliable decision than an erasure. To better realize these statements, it is useful to consider the results reported in Fig. 4, which refer to a binary PSK (BPSK) modulation with alphabet $\{1,-1\}$ and a class-A channel characterized by $A=10^{-1}, \Gamma=10^{-1}$, and an SNR of $0 \mathrm{~dB}$. In Fig. 4, it is shown how the log-likelihood ratio (LLR), that is the natural logarithm of the ratio between the metric corresponding to the hypothesis $\tilde{c}_{k}=1$ and that corresponding to the hypothesis $\tilde{c}_{k}=-1$, varies with respect to the received sample $y_{k}$ when different metrics are considered. The behavior of the optimal metric exhibits two key points, namely the need for producing very low-magnitude LLRs when the received sample is far away from the constellation, that is when the presence of interfering impulses is very likely, and the need for exploiting the imaginary component of $y_{k}$-unlike the AWGN channels, the real and imaginary components of the class-A noise are not independent. Fig. 4 definitely proves that the proposed metric, here implemented with $\Delta=10^{-3}$, approximate this behavior much better than the considered alternatives. In particular, both the AWGN metric and the SL metric, here implemented with $V_{T}=1.3$, cannot exploit the imaginary component of $y_{k}$ (thus not reported in Fig. 4) and dramatically fail in producing low-magnitude LLRs when the presence of interfering impulses is very likely. In conclusion, it is easy to predict that the proposed metric will significantly outperform them when systems requiring high-quality softoutput detection are considered. On the other hand, the generation of erasure decisions (that is null LLRs in Fig. 4) makes the proposed metric less suitable for hard-output detection.

We now discuss the choice of the threshold parameter $\Delta$, which is crucial for the performance of the proposed metric. Following the arguments in [8], one can derive a rule of thumb for choosing the value of $\Delta$ given the statistics of the impulse noise. On the other hand, extensive computer simulations, some of which are reported in Section V, show that the proposed metric is very robust, and that values of $\Delta$ in the order of $10^{-3}$ result effective irrespectively of the actual statistics of the impulse noise, provided that a powerful

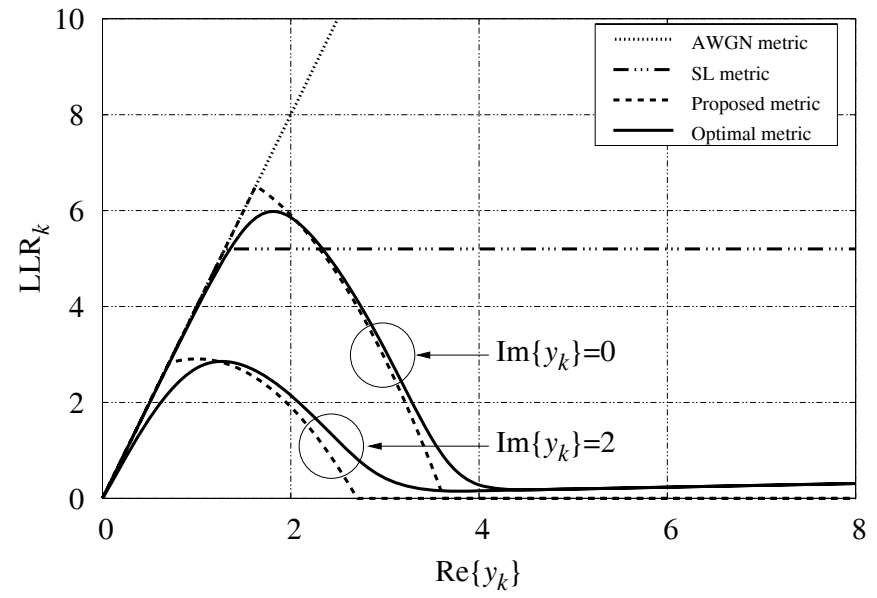

Fig. 4. Log-likelihood ratios for a channel characterized by $A=10^{-1}$, $\Gamma=10^{-1}$, and SNR $=0 \mathrm{~dB}$.

channel code is adopted. Such a robustness is due to the fact that the value of $\Delta$ does not affect the key features required for good soft-output detection, namely the generation of lowmagnitude LLRs when the presence of interfering impulses is very likely, and the capability of exploiting that the real and imaginary components of the received samples are not independent.

\section{Simulation Results}

In this section, the performance of the described detection schemes is assessed by means of computer simulations. The reported results refer to QPSK transmissions over channels characterized by different statistics of the impulse noise. A $(3,6)$-regular LDPC code of rate $1 / 2$ is applied to sequences of 2000 information bits. At the receiver side, the LDPC decoder performs 40 self-iterations before producing the decisions on the information bits [10]. The iterative process can also stop before the $40^{\text {th }}$ iteration if, by checking the code syndrome, a valid codeword is found.

We first consider a channel with impulse noise characterized by $A=10^{-1}$ and $\Gamma=10^{-1}$. Fig. 5 shows the performance of the system when different metrics are used, in terms of BER versus SNR. As a comparison, the performance over an AWGN channel is also reported. Although, according to (6), the impulse noise increases the power of the overall noise of about $10 \mathrm{~dB}$ with respect to the AWGN channel, the optimal metric provides a performance degradation lower than $1.5 \mathrm{~dB}$, canceling out the greatest part of the impulse noise thanks to the powerful coding scheme. We notice that the proposed metric, here implemented with $\Delta=10^{-3}$, ensures the same performance as the optimal one, resulting the most convenient performance/complexity tradeoff. Let us point out that the saturation to the minimum threshold $\Delta$, which actually is the only difference between the proposed metric and the AWGN metric, provides a gain of more than $12 \mathrm{~dB}$ at the expense of a practically null increase in complexity. On the other hand, the simulation results also confirm the conjectures carried out in Section IV-B on the ineffectiveness of the SL metric when employed in systems requiring high-quality soft-output detection. In fact, although the threshold value $V_{T}$ has been 


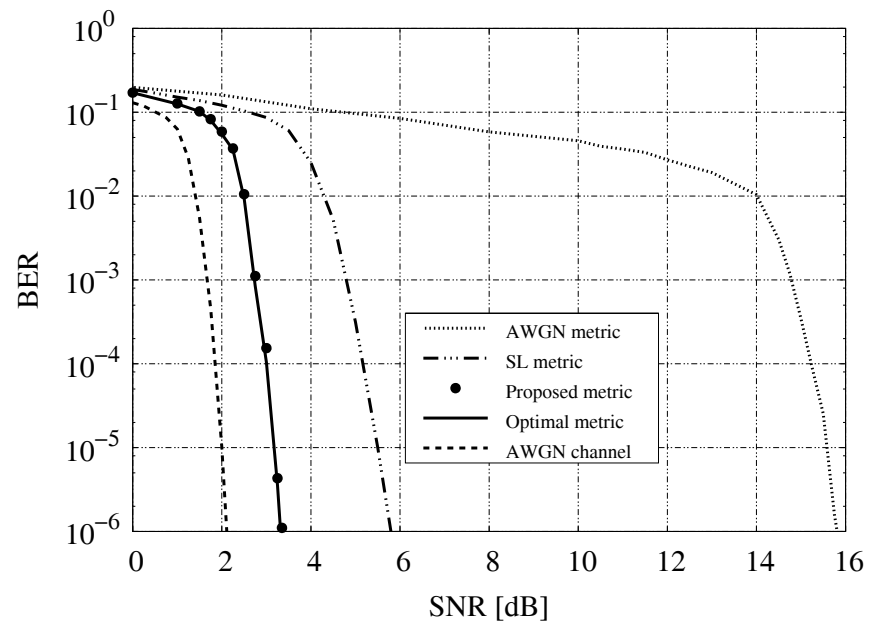

Fig. 5. Performance over a class-A channel characterized by $A=10^{-1}$ and $\Gamma=10^{-1}$.

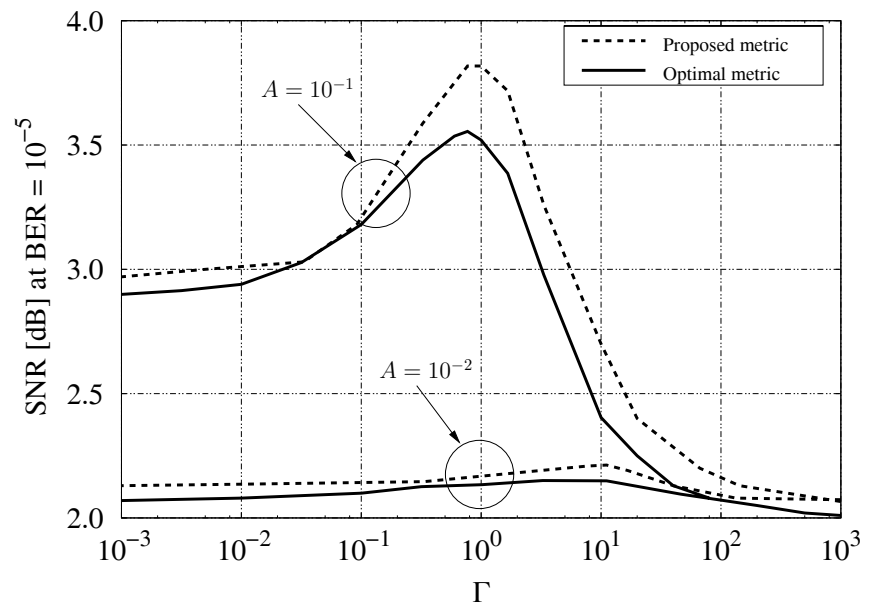

Fig. 6. Performance over channels with different parametrizations of the impulse noise.

optimized for each value of the SNR, we notice that the SL metric exhibits a performance degradation larger than $2 \mathrm{~dB}$ with respect to the proposed one.

Fig. 6 shows the values of the SNR corresponding to a BER equal to $10^{-5}$ when different statistics of the impulse noise are considered. The performance of the optimal metric and that of the proposed one working with $\Delta=10^{-3}$ are compared. We remark the robustness of the proposed metric, which provides a negligible performance degradation with respect to the ideal benchmark irrespectively of the statistics of the impulse noise. In practice, when the proposed metric is adopted, we can just set $\Delta=10^{-3}$ and there is no need for information on the values of $A$ and $\Gamma$.

This fact, together with the very low computational complexity, definitely makes the proposed solution the most convenient one. The same conclusion holds even when a simplified model is assumed for the impulse noise [7]. Moreover, it is worth to notice that the results reported in Fig. 6 greatly agree with the theoretical discussion carried out in Section III.

In particular, it is confirmed that the impulse noise, irrespectively of its power, can be practically canceled out when the impulsive index is small enough (with the same LDPC code, over an AWGN channel, a BER equal to $10^{-5}$ is achieved when the value of the SNR is about $2 \mathrm{~dB}$ ), and that there exists a value $\Gamma_{0}$ such that, for $\Gamma<\Gamma_{0}$, the system can take advantage of a larger power of the impulse noise.

\section{CONCLUSIONS}

The performance of communication systems over channels impaired by impulse noise has been analyzed. We have discussed the ultimate performance limits of these systems by exploiting information-theoretical arguments, and presented a detection metric that, besides being characterized by a minimal computational complexity, does not require the knowledge of the statistics of the impulse noise. When combined with powerful channel codes, the proposed scheme has been shown to perform practically as the ideal one, much better than the classical soft-limiting detectors, and fairly close to the theoretical limit.

\section{REFERENCES}

[1] D. Middleton, "Statistical-physical model of electromagnetic interference," IEEE Trans. Electromagn. Compat., vol. 19, no. 3, pp. 106-126, Aug. 1977.

[2] A. D. Spaulding and D. Middleton, "Optimum reception in an impulsive interference environment-part I: coherent detection," IEEE Trans. Commun., vol. 25, pp. 910-923, Sept. 1977.

[3] D. Umehara, H. Yamaguchi, and Y. Morihiro, "Turbo decoding in impulsive noise environment," in Proc. IEEE Global Telecommun. Conf., Dallas, Texas, Nov./Dec. 2004.

[4] H. Nakagawa, D. Umehara, S. Denno, and Y. Morihiro, "A decoding for low density parity check codes over impulsive noise channels," in Proc. IEEE Intl. Symp. on Power Line Commun. Its Appl., Vancouver, Canada, Apr. 2005.

[5] S. M. Zabin and H. V. Poor, "Efficient estimation of class A noise parameter via the EM algorithm," IEEE Trans. Inform. Theory, vol. 37, pp. 60-72, Jan. 1991.

[6] J. Mitra and L. Lampe, "Robust decoding for channels with impulse noise," in Proc. IEEE Global Telecommun. Conf., San Francisco, CA, Nov./Dec. 2006.

[7] D. Fertonani and G. Colavolpe, "A simplified metric for soft-output detection in the presence of impulse noise," in Proc. IEEE Intl. Symp. on Power Line Commun. Its Appl., Pisa, Italy, pp. 121-126, Mar. 2007.

[8] D. Fertonani and G. Colavolpe, "Theoretical limits and practical detection schemes for channels affected by class-A impulse noise," in Proc. IEEE Global Telecommun. Conf., Washington, DC, USA, pp. 146150, Nov. 2007.

[9] S. Benedetto, D. Divsalar, G. Montorsi, and F. Pollara, "Serial concatenation of interleaved codes: performance analysis, design, and iterative decoding," IEEE Trans. Inform. Theory, vol. 44, pp. 909-926, May 1998.

[10] T. Richardson and R. Urbanke, "The capacity of low density parity check codes under message passing decoding," IEEE Trans. Inform. Theory, vol. 47, pp. 599-618, Feb. 2001

[11] G. L. Stüber, "Soft-limiter receivers for coded DS/DPSK systems," IEEE Trans. Commun., vol. 38, pp. 46-53, Jan. 1990.

[12] T. M. Cover and J. A. Thomas, Elements of Information Theory. New York: John Wiley \& Sons, Inc., 1991.

[13] A. Goldsmith and P. Varaiya, "Capacity, mutual information and coding for finite-state Markov channels," IEEE Trans. Inform. Theory, vol. 42, pp. 868-886, May 1996. 